\title{
THE BADLY BEHAVED PRODUCTION FUNCTION: COMMENT
}

\author{
Michael Bruno, Edwin Burmeister, and Eytan Sheshinski
}

In their recent article Joan Robinson and K. A. Naqvi provide an example which they think ". . . conclusively disproves the notion that the number of switches cannot be more than the number of capital goods in the system." 1 This assertion relates to a theorem recently proved by us. ${ }^{2}$ A glance at their own example shows, however, that their conclusion is completely unwarranted.

Consider their technology or "book of blue prints" which is composed of techniques $A$ and $A_{a}$. The technology consists of three capital goods, wheat, iron, and aluminum, which we will designate by the indices 1,2 , and 3 , respectively. As usual, $a_{i j}$ is the input coefficient of commodity $i$ for the production of good $j$, and the index $o$ denotes a labor input. Thus the technology for the RobinsonNaqvi "counterexample" must be written as follows:

\section{Technique $A$}

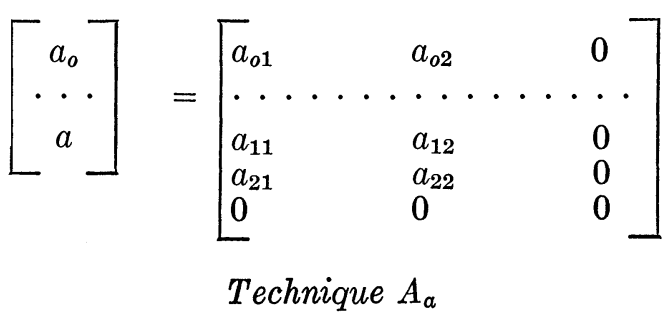

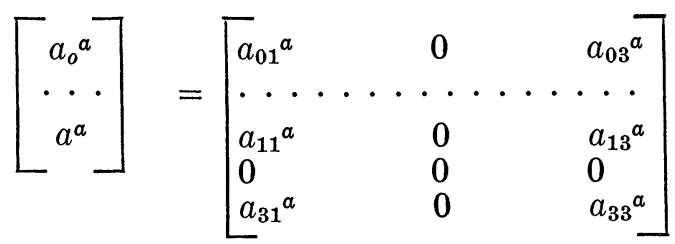

There exists a factor-price frontier for this technology which gives the wage rate in terms of the price of wheat as a function of

1. Joan Robinson, and K. A. Naqvi, "The Badly Behaved Production Function," this Journal LXXXI (Nov. 1967), 590-91.

2. Michael Bruno, Edwin Burmeister, and Eytan Sheshinski, this Journal LXXX (Nov. 1966). On page 532 we state that there may exist up to $n$ switching points between any two nxn technique matrixes. It is true that there may exist more than $n$ switching points if the technology consists of more than two alternative techniques. 
the profit or interest rate in alternative steady states, i.e., there is a function

$$
w=\frac{W}{P_{1}}=f(r) .
$$

Of course, $f(r)$ is the outer-envelope of the factor-price curves for the techniques $A$ and $A_{a}$. Since there are three capital goods, the latter curves may intersect up to (but no more than) three times, as asserted by our theorem. Consequently the Robinson-Naqvi "counterexample" is completely invalid.

Finally, let us raise one more question. What is the precise meaning of the term "production function" in their title "The Badly Behaved Production Function"? What we mean by a production function is output of commodity $j$ given as a function of the capital inputs $K_{1 j}, \ldots, K_{n j}$ and the labor input $L_{j}$, i.e.,

$$
Q_{j}=F^{j}\left(K_{1 j}, \ldots, K_{n j}, L_{j}\right) \quad(j=1, \ldots, n) .
$$

In a linear technology with one technique matrix of technical constants defined by

$$
\begin{array}{lll}
\text { and } & a_{i j}=K_{i j} / Q_{j} & (i, j=1, \ldots, n) \\
& a_{o j}=L_{j} / Q_{j} & (j=1, \ldots, n),
\end{array}
$$

the functions $F$ are of the familiar form

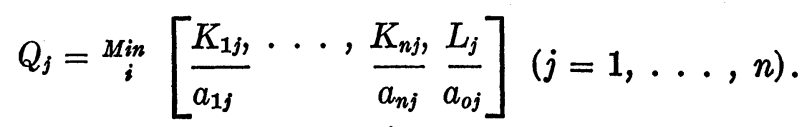

Certainly these production functions are not "badly behaved," but rather it is the technology, which is composed of the entire set of alternative technique matrixes $\left[\begin{array}{c}a_{o} \\ \cdot \vec{a}\end{array}\right],\left[\begin{array}{c}b_{o} \\ \dot{b}\end{array}\right], \ldots$, etc., which may exhibit paradoxical behavior. What Robinson and Naqvi probably mean is that a function with capital "jelly" as an argument may exhibit paradoxical behavior. But this is just another example of the familiar "aggregation problem." We simply prefer not to call it a production function at all.

HEBREW UNIVERSITY

University of Pennsyluania

HEBREW UNIVERSITY 